Kragujevac Journal of Mathematics

Volume 45(2) (2021), Pages 299-307.

\title{
ON THE HARMONIC INDEX AND THE SIGNLESS LAPLACIAN SPECTRAL RADIUS OF GRAPHS
}

\author{
HANYUAN DENG ${ }^{1}$, TOMÁŠ VETRÍK ${ }^{2}$, AND SELVARAJ BALACHANDRAN ${ }^{2,3}$
}

ABSTRACT. The harmonic index of a conected graph $G$ is defined as $H(G)=$ $\sum_{u v \in E(G)} \frac{2}{d(u)+d(v)}$, where $E(G)$ is the edge set of $G, d(u)$ and $d(v)$ are the degrees of vertices $u$ and $v$, respectively. The spectral radius of a square matrix $M$ is the maximum among the absolute values of the eigenvalues of $M$. Let $q(G)$ be the spectral radius of the signless Laplacian matrix $Q(G)=D(G)+A(G)$, where $D(G)$ is the diagonal matrix having degrees of the vertices on the main diagonal and $A(G)$ is the $(0,1)$ adjacency matrix of $G$. The harmonic index of a graph $G$ and the spectral radius of the matrix $Q(G)$ have been extensively studied. We investigate the relationship between the harmonic index of a graph $G$ and the spectral radius of the matrix $Q(G)$. We prove that for a connected graph $G$ with $n$ vertices, we have

$$
\frac{q(G)}{H(G)} \leq \begin{cases}\frac{n^{2}}{2(n-1)}, & \text { if } n \geq 6, \\ \frac{16}{5}, & \text { if } n=5, \\ 3, & \text { if } n=4,\end{cases}
$$

and the bounds are best possible.

\section{INTRODUCTION}

A lot of research has been done on topological indices due to their chemical importance. Chemical-based experiments show that there is a strong relationship between the properties of chemical compounds and their molecular structures. Topological indices are used for modelling properties of chemical compounds and biological activities in chemistry, biochemistry and nanotechnology. We study the harmonic index which is one of the most known topological indices.

Key words and phrases. Harmonic index, spectral radius, eigenvalue, signless Laplacian matrix. 2010 Mathematics Subject Classification. Primary: 05C50. Secondary: 05C35.

DOI 10.46793/KgJMat2102.299D

Received: April 30, 2018.

Accepted: December 11, 2018. 
Let $G$ be a simple connected graph with vertex set $V(G)$ and edge set $E(G)$. The degree of a vertex $v \in V(G), d(v)$, is the number of edges incident with $v$. A tree is a connected graph containing no cycles and a unicyclic graph is a connected graph containing exactly one cycle. A bicyclic graph is a connected graph $G$ having $n+1$ edges where $n$ is the number of vertices of $G$. Let us denote the complete graph, the star and the path having $n$ vertices by $K_{n}, S_{n}$ and $P_{n}$, respectively.

Let $e_{1}, e_{2}, \ldots, e_{k} \in E(G)$. We denote by $G-\left\{e_{1}, e_{2}, \ldots, e_{k}\right\}$ the graph with vertex set $V(G)$ and edge set $E(G) \backslash\left\{e_{1}, e_{2}, \ldots, e_{k}\right\}$. An edge-induced subgraph $G\left[e_{1}, e_{2}, \ldots, e_{k}\right]$ is a subgraph of $G$ which consists of the edges $e_{1}, e_{2}, \ldots, e_{k}$ and vertices incident with $e_{1}, e_{2}, \ldots, e_{k}$.

The spectral radius of a square matrix $M$ is the maximum among the absolute values of the eigenvalues of $M$. Let $q(G)$ be the spectral radius of the signless Laplacian matrix $Q(G)=D(G)+A(G)$, where $D(G)$ is the diagonal matrix having degrees of the vertices on the main diagonal and $A(G)$ is the $(0,1)$ adjacency matrix of $G$. We denote the spectral radius (of the adjacency matrix $A(G)$ ) of a graph $G$ by $\lambda(G)$.

The Randić index of a graph $G$ is defined as

$$
R(G)=\sum_{u v \in E(G)} \frac{1}{\sqrt{d(u) d(v)}}
$$

This topological index has been successfully related to chemical and physical properties of organic molecules, and become one of the most important molecular descriptors. The Randić index was introduced by Randić [16] and generalized by Bollobás and Erdős [3]. Using the AutoGraphiX2 system, Aouchiche, Hansen and Zheng [1,2] studied lower and upper bounds on $R(G) \oplus i(G)$ in terms of the number of vertices of $G$, where $i(G)$ is one of the following invariants: the maximum, minimum and average degree, diameter, girth, algebraic and vertex connectivity, matching number and the spectral radius of $G$, and $\oplus$ denotes one of the four operations,,$+- \times, /$.

The harmonic index

$$
H(G)=\sum_{u v \in E(G)} \frac{2}{d(u)+d(v)}
$$

of a graph $G$ was introduced by Fajtlowicz [8]. Hansen and Vukicević [12] studied the connection between the Randić index and the chromatic number of graphs. Deng et al. [6] considered the relation connecting the harmonic index and the chromatic number and strengthened the result relating the Randic index and the chromatic number conjectured by the system AutoGraphiX and proved in [12]. Favaron, Mahéo and Saclé [9] considered the relationship between the harmonic index and eigenvalues of a graph. 
Using the AutoGraphiX system, Hansen and Lucas [11] gave a conjecture saying that if $G$ is a connected graph having $n \geq 4$ vertices, then

$$
\frac{q(G)}{R(G)} \leq \begin{cases}\frac{4 n-4}{n}, & \text { if } 4 \leq n \leq 12 \\ \frac{n}{\sqrt{n-1}}, & \text { if } n \geq 13\end{cases}
$$

with equality if and only if $G$ is $K_{n}$ for $4 \leq n \leq 12$ and $G$ is $S_{n}$ for $n \geq 13$. Recently, Ning and Peng [15] solved this conjecture.

Motivated by the work of [15] we study the relationship between the harmonic index $H(G)$ of a graph $G$ and the spectral radius of the signless Laplacian matrix $Q(G)$. In particular, we prove the following theorem.

Theorem 1.1. Let $G$ be a connected graph having $n$ vertices. Then

$$
\frac{q(G)}{H(G)} \leq \begin{cases}\frac{n^{2}}{2(n-1)}, & \text { if } n \geq 6, \\ \frac{16}{5}, & \text { if } n=5, \\ 3 & \text { if } n=4,\end{cases}
$$

with equality if and only if $G$ is $S_{n}$ for $n \geq 6$ and $G$ is $K_{n}$ for $4 \leq n \leq 5$.

\section{Preliminaries}

In this section, we present known results, which will be used in the proofs of our theorems. Upper bounds on the spectral radius of the signless Laplacian matrix and the adjacency matrix of a graph were given in [10] and [13], respectively.

Lemma 2.1 ([10]). Let $G$ be a connected graph with $n$ vertices, $m$ edges and let $q(G)$ be the spectral radius of the signless Laplacian matrix of $G$. Then

$$
q(G) \leq \frac{2 m}{n-1}+n-2,
$$

with equality if and only if $G$ is $K_{n}$ or $S_{n}$.

Lemma $2.2([13])$. Let $G$ be a connected graph $G$ with $n$ vertices, $m$ edges and let $\lambda(G)$ be the spectral radius of the adjacency matrix of $G$. Then

$$
\lambda(G) \leq \sqrt{2 m-n+1},
$$

with equality if and only if $G$ is $K_{n}$ or $S_{n}$.

Let us present three lower bounds on the harmonic index $H(G)$ of a graph $G$ for general graphs, unicyclic graphs and bicyclic graphs.

Lemma $2.3([5])$. Let $G$ be a connected graph with $n$ vertices and $m$ edges. Then

$$
H(G) \geq \frac{2 m^{2}}{n \lambda(G)^{2}} .
$$


Lemma $2.4([7,14,17])$. Let $G$ be a unicyclic graph with $n \geq 3$ vertices. Then

$$
H(G) \geq \frac{5 n^{2}+n-12}{2 n(n+1)} .
$$

Lemma 2.5 ([7,14]). Let $G$ be a bicyclic graph with $n \geq 4$ vertices. Then

$$
H(G) \geq \frac{14}{5}-\frac{2 n^{2}+14 n+16}{n(n+1)(n+2)} .
$$

\section{Results}

First we consider graphs with $n \geq 6$ vertices, $m$ edges and the harmonic index at least $\frac{2(n-1)}{n}+\frac{4(m-n+1)}{n^{2}}$.

Theorem 3.1. Let $G$ be a connected graph with $n \geq 6$ vertices and $m$ edges. If $H(G) \geq \frac{2(n-1)}{n}+\frac{4(m-n+1)}{n^{2}}$, then

$$
\frac{q(G)}{H(G)} \leq \frac{n^{2}}{2(n-1)}
$$

with equality if and only if $G$ is $S_{n}$.

Proof. Let $H(G) \geq \frac{2(n-1)}{n}+\frac{4(m-n+1)}{n^{2}}$. By Lemma 2.1, we have $q(G) \leq \frac{2 m}{n-1}+n-2$, with equality if and only if $G$ is either $K_{n}$ or $S_{n}$. Thus,

$$
\frac{q(G)}{H(G)} \leq \frac{\frac{2 m}{n-1}+n-2}{\frac{2(n-1)}{n}+\frac{4(m-n+1)}{n^{2}}}=\frac{\left(\frac{2(n-1)}{n}+\frac{4(m-n+1)}{n^{2}}\right) \frac{n^{2}}{2(n-1)}}{\frac{2(n-1)}{n}+\frac{4(m-n+1)}{n^{2}}}=\frac{n^{2}}{2(n-1)} .
$$

Note that for $K_{n}$ we have $m=\frac{n(n-1)}{2}$ and for this value we get $\frac{2(n-1)}{n}+\frac{4(m-n+1)}{n^{2}}=$ $4\left(\frac{n-1}{n}\right)^{2}$. Since $H\left(K_{n}\right)=\frac{n}{2}>4\left(\frac{n-1}{n}\right)^{2}$ for every $n \geq 6$, we obtain $\frac{q\left(K_{n}\right)}{H\left(K_{n}\right)}<\frac{n^{n^{2}}}{2(n-1)}$.

For the graph $S_{n}$ we have $m=n-1$. Since $H\left(S_{n}\right)=\frac{2(n-1)}{n}=\frac{2(n-1)}{n}+\frac{4(m-n+1)}{n^{2}}$ for $m=n-1$, we get $\frac{q\left(S_{n}\right)}{H\left(S_{n}\right)}=\frac{n^{2}}{2(n-1)}$, which implies that $\frac{q(G)}{H(G)} \leq \frac{n^{2}}{2(n-1)}$, with equality if and only if $G$ is $S_{n}$.

Let us show that the main result holds for trees and graphs satisfying the inequality $m \geq n+1+\frac{6}{n-4}$.

Theorem 3.2. Let $G$ be a connected graph with $n \geq 6$ vertices and $m$ edges.

(i) If $m=n-1$, then $\frac{q(G)}{H(G)} \leq \frac{n^{2}}{2(n-1)}$, with equality if and only if $G$ is $S_{n}$.

(ii) If $m \geq n+1+\frac{6}{n-4}$, then $\frac{q(G)}{H(G)}<\frac{n^{2}}{2(n-1)}$.

(iii) If $n=6,7$ and $m=10$, then $\frac{q(G)}{H(G)}<\frac{n^{2}}{2(n-1)}$.

Proof. By Lemmas 2.2 and 2.3 we have $H(G) \geq \frac{2 m^{2}}{n \lambda^{2}} \geq \frac{2 m^{2}}{n(2 m-n+1)}$. Let $f(m)=$ $H(G)-\frac{2(n-1)}{n}-\frac{4(m-n+1)}{n^{2}}$. Then

$$
f(m) \geq \frac{2 m^{2}}{n(2 m-n+1)}-\frac{2(n-1)}{n}-\frac{4(m-n+1)}{n^{2}}=\frac{2 \times g(m)}{n^{2}(2 m-n+1)},
$$


where $g(m)=(m-n+1)\left[(n-4) m-\left(n^{2}-3 n+2\right)\right]$.

(i) If $m=n-1$, then $g(m)=0$ which implies that $f(m) \geq 0$ and $H(G) \geq$ $\frac{2(n-1)}{n}+\frac{4(m-n+1)}{n^{2}}$. From Theorem 3.1 we get $\frac{q(G)}{H(G)} \leq \frac{n^{2}}{2(n-1)}$, with equality if and only if $G$ is $S_{n}$.

(ii) If $m \geq n+1+\frac{6}{n-4}=\frac{n^{2}-3 n+2}{n-4}$, then $(n-4) m-\left(n^{2}-3 n+2\right) \geq 0$. This implies that $g(m) \geq 0$ and $f(m) \geq 0$. Thus, $H(G) \geq \frac{2(n-1)}{n}+\frac{4(m-n+1)}{n^{2}}$. Since $G$ is not $S_{n}$, from Theorem 3.1, we get $\frac{q(G)}{H(G)}<\frac{n^{2}}{2(n-1)}$.

(iii) If $n=6$ and $m=10$, or $n=7$ and $m=10$, or $n=10$ and $m=12$, then $g(m)=0$. Thus $f(m) \geq 0$ and $H(G) \geq \frac{2(n-1)}{n}+\frac{4(m-n+1)}{n^{2}}$. Since $G$ is not $S_{n}$, from Theorem 3.1 we get $\frac{q(G)}{H(G)}<\frac{n^{2}}{2(n-1)}$.

The following two results solve our problem for every $m=n$ and $m=n+1$, respectively.

Theorem 3.3. Let $G$ be a connected graph with $n \geq 6$ vertices and $m=n$ edges. Then

$$
\frac{q(G)}{H(G)}<\frac{n^{2}}{2(n-1)} .
$$

Proof. If $m=n$, then $G$ is a unicyclic graph and by Lemma 2.4 we have $H(G) \geq$ $\frac{5 n^{2}+n-12}{2 n(n+1)}$. It can be checked that $\frac{5 n^{2}+n-12}{2 n(n+1)}>\frac{2(n-1)}{n}+\frac{4}{n^{2}}$. Then from Theorem 3.1 we obtain $\frac{q(G)}{H(G)}<\frac{n^{2}}{2(n-1)}$.

Theorem 3.4. Let $G$ be a connected graph with $n \geq 6$ and $m=n+1$ edges. Then

$$
\frac{q(G)}{H(G)}<\frac{n^{2}}{2(n-1)}
$$

Proof. If $m=n+1$, then $G$ is a bicyclic graph and by Lemma 2.5 we have $H(G) \geq$ $\frac{14}{5}-\frac{2 n^{2}+14 n+16}{n(n+1)(n+2)}$. It can be checked that $\frac{14}{5}-\frac{2 n^{2}+14 n+16}{n(n+1)(n+2)}>\frac{2(n-1)}{n}+\frac{8}{n^{2}}$. Then, from Theorem 3.1, we obtain $\frac{q(G)}{H(G)}<\frac{n^{2}}{2(n-1)}$.

From Theorems 3.2, 3.3 and 3.4 we obtain the best possible bound on $\frac{q(G)}{H(G)}$ for graphs $G$ having $n \geq 10$ vertices.

Corollary 3.1. Let $G$ be a connected graph with $n \geq 10$ vertices. Then

with equality if and only if $G$ is $S_{n}$.

$$
\frac{q(G)}{H(G)} \leq \frac{n^{2}}{2(n-1)},
$$

Proof. Since $n+2 \geq n+1+\frac{6}{n-4}$ for $n \geq 10$, by Theorem 3.2 (ii), $\frac{q(G)}{H(G)}<\frac{n^{2}}{2(n-1)}$ for every $n \geq 10$ and $m \geq n+2$. By Theorems 3.3 and 3.4, $\frac{q(G)}{H(G)}<\frac{n^{2}}{2(n-1)}$ for graphs $G$ such that $m=n$ and $m=n+1$, and by Theorem 3.2 (i), $\frac{q(G)}{H(G)} \leq \frac{n^{2}}{2(n-1)}$ for graphs $G$ such that $m=n-1$ with equality if and only if $G$ is $S_{n}$. 
From Theorems 3.2, 3.3 and 3.4 we also know that if $6 \leq n \leq 9$, then the only cases which remain unsolved are:

(i) $n=6$ and $m=8,9$;

(ii) $n=7$ and $m=9$;

(iii) $n=8$ and $m=10$;

(iv) $n=9$ and $m=11$.

For this purpose we present results on the spectral radius of connected graphs with $n$ vertices and $n+2 \leq m \leq n+3$ edges. From [4, Theorems 3.2 and 3.3] and their proofs we obtain the following lemma.

Lemma 3.1 ([4]). The maximum spectral radius $\lambda(G)$ of a connected graph $G$ with $n \geq 4$ vertices and $m$ edges is the maximum root of

(i) $\varphi_{1}(\lambda)=\lambda^{3}-2 \lambda^{2}-(n-1) \lambda+2(n-4)$ if $m=n+2$;

(ii) $\varphi_{2}(\lambda)=\lambda^{4}-(n+3) \lambda^{2}-8 \lambda+4(n-6)$ and

$$
\varphi_{3}(\lambda)=\lambda^{6}-(n+3) \lambda^{4}-10 \lambda^{3}+(4 n-21) \lambda^{2}+(2 n-8) \lambda-(n-5) \text { if } m=n+3 .
$$

We use Lemma 3.1 in the proof of Theorem 3.5.

Theorem 3.5. Let $G$ be a connected graph with $n$ vertices and $m$ edges. If

(i) $n=6$ and $m=8,9$;

(ii) $n=7$ and $m=9$;

(iii) $n=8$ and $m=10$;

(iv) $n=9$ and $m=11$,

then $\frac{q(G)}{H(G)}<\frac{n^{2}}{2(n-1)}$.

Proof. By Lemma 3.1, we can calculate the upper bounds on the maximum spectral radius $\lambda$. We have $\lambda<3.1775$ if $n=6$ and $m=8, \lambda<3.274$ if $n=7$ and $m=9$, $\lambda<3.373$ if $n=8$ and $m=10, \lambda<3.475$ if $n=9$ and $m=11, \lambda<3.404$ if $n=6$ and $m=9$.

Thus, from Lemma 2.3 we obtain the lower bounds on the harmonic index of G. $H(G)>2.11294$ if $n=6$ and $m=8, H(G)>2.15903$ if $n=7$ and $m=9$, $H(G)>2.19739$ if $n=8$ and $m=10, H(G)>2.22671$ if $n=9$ and $m=11$, $H(G)>2.33015$ if $n=6$ and $m=9$.

By Lemma 2.1, we obtain upper bounds on $q(G)$. We have $q(G) \leq \frac{36}{5}$ if $n=6$ and $m=8, q(G) \leq 8$ if $n=7$ and $m=9, q(G) \leq \frac{62}{7}$ if $n=8$ and $m=10, q(G) \leq \frac{39}{4}$ if $n=9$ and $m=11, q(G) \leq \frac{38}{5}$ if $n=6$ and $m=9$.

It is easy to verify that $\frac{q(G)}{H(G)}<\frac{n^{2}}{2(n-1)}$ for all these cases.

From Theorems 3.2, 3.3, 3.4 and 3.5 we get Corollary 3.2.

Corollary 3.2. Let $G$ be a connected graph with $n$ vertices where $6 \leq n \leq 9$. Then

$$
\frac{q(G)}{H(G)} \leq \frac{n^{2}}{2(n-1)}
$$

with equality if and only if $G$ is $S_{n}$. 
It remains to find upper bounds on $\frac{q(G)}{H(G)}$ for graphs $G$ having $n \leq 5$ vertices. For $n=3$ there are only two non-isomorphic graphs: $K_{3}$ and $K_{3}-\{e\}$, where $e \in E\left(K_{3}\right)$. We have $H\left(K_{3}\right)=\frac{3}{2}$ and by Lemma $2.1, q\left(K_{3}\right)=4$, thus $\frac{q\left(K_{3}\right)}{H\left(K_{3}\right)}=\frac{8}{3}$. For $K_{3}-\{e\}$ we obtain $H\left(K_{3}-\{e\}\right)=\frac{4}{3}$ and by Lemma 2.1, $q\left(K_{3}-\{e\}\right) \leq 3$, so $\frac{q\left(K_{3}-\{e\}\right)}{H\left(K_{3}-\{e\}\right)} \leq \frac{9}{4}$. Hence $\frac{q(G)}{H(G)} \leq \frac{8}{3}$ for any graph $G$ having 3 vertices with equality if and only if $G$ is $K_{3}$.

Let us present bounds for graphs having 4 and 5 vertices.

Theorem 3.6. Let $G$ be a connected graph with 4 vertices. Then

$$
\frac{q(G)}{H(G)} \leq 3
$$

with equality if and only if $G$ is $K_{4}$.

Proof. The only graph with 4 vertices and 6 edges is $K_{4}$, and the only graph with 4 vertices and 5 edges is $K_{4}-\{e\}$. Since $H\left(K_{4}\right)=2$ and $q\left(K_{4}\right)=6$ (by Lemma 2.1), we get $\frac{q\left(K_{4}\right)}{H\left(K_{4}\right)}=3$.

For $K_{4}-\{e\}$ where $e \in E\left(K_{4}\right)$, we obtain $H\left(K_{4}-\{e\}\right)=\frac{29}{15}$, and from Lemma 2.1 we have $q\left(K_{4}-\{e\}\right) \leq \frac{16}{3}$, which gives $\frac{q\left(K_{4}-\{e\}\right)}{H\left(K_{4}-\{e\}\right)} \leq \frac{80}{29}<3$.

We have two non-isomorphic graphs for $m=4$, namely $C_{4}$ and $S_{4}+\{e\}$. We get $H\left(C_{4}\right)=2$ and $q\left(C_{4}\right) \leq \frac{14}{3}$ (by Lemma 2.1), so $\frac{q\left(C_{4}\right)}{H\left(C_{4}\right)} \leq \frac{7}{3}<3$. Similarly, $H\left(S_{4}+\{e\}\right)=\frac{9}{5}$ and $q\left(S_{4}+\{e\}\right) \leq \frac{14}{3}$, thus $\frac{q\left(S_{4}+\{e\}\right)}{H\left(S_{4}+\{e\}\right)} \leq \frac{70}{27}<3$.

There are two non-isomorphic graphs for $m=3$, namely $S_{4}$ and $P_{4}$. We have $H\left(S_{4}\right)=\frac{3}{2}$ and $q\left(S_{4}\right)=4$ (by Lemma 2.1), thus $\frac{q\left(S_{4}\right)}{H\left(S_{4}\right)}=\frac{8}{3}$. Similarly, $H\left(P_{4}\right)=\frac{11}{6}$ and $q\left(P_{4}\right) \leq 4$, hence $\frac{q\left(P_{4}\right)}{H\left(P_{4}\right)} \leq \frac{24}{11}<3$.

Theorem 3.7. Let $G$ be a connected graph with 5 vertices. Then

$$
\frac{q(G)}{H(G)} \leq \frac{16}{5}
$$

with equality if and only if $G$ is $K_{5}$.

Proof. We consider the cases $m=7,8,9,10$. The only graph with 5 vertices and 10 edges is $K_{5}$. Since $H\left(K_{5}\right)=\frac{5}{2}$ and $q\left(K_{5}\right)=8$ (by Lemma 2.1), we get $\frac{q\left(K_{5}\right)}{H\left(K_{5}\right)}=\frac{16}{5}$.

The only graph with 5 vertices and 9 edges is $K_{5}-\{e\}$ where $e \in E\left(K_{5}\right)$. We have $H\left(K_{5}-\{e\}\right)=\frac{69}{28}$ and from Lemma 2.1 we obtain $q\left(K_{5}-e\right) \leq \frac{15}{2}$, which gives $\frac{q\left(K_{5}-e\right)}{H\left(K_{5}-e\right)} \leq \frac{210}{69}<\frac{16}{5}$.

For $m=8$ we have $G=K_{5}-\left\{e_{1}, e_{2}\right\}$ where $e_{1}, e_{2} \in E\left(K_{5}\right)$. There are two nonisomorphic graphs having 8 edges. If $e_{1}$ and $e_{2}$ are adjacent, then $H\left(K_{5}-\left\{e_{1}, e_{2}\right\}\right)=\frac{67}{28}$, and if $e_{1}$ and $e_{2}$ are not adjacent, then $H\left(K_{5}-\left\{e_{1}, e_{2}\right\}\right)=\frac{52}{21}$. So, $H\left(K_{5}-\left\{e_{1}, e_{2}\right\}\right) \geq \frac{67}{28}$ 
and from Lemma 2.1 we obtain $q\left(K_{5}-\left\{e_{1}, e_{2}\right\}\right) \leq 7$, which gives

$$
\frac{q\left(K_{5}-\left\{e_{1}, e_{2}\right\}\right)}{H\left(K_{5}-\left\{e_{1}, e_{2}\right\}\right)} \leq \frac{196}{67}<\frac{16}{5} .
$$

For $m=7$ we have $G=K_{5}-\left\{e_{1}, e_{2}, e_{3}\right\}$ where $e_{1}, e_{2}, e_{3} \in E\left(K_{5}\right)$. There are four non-isomorphic graphs having 7 edges. If $G\left[e_{1}, e_{2}, e_{3}\right]$ is $K_{3}$, then $H\left(K_{5}-\left\{e_{1}, e_{2}, e_{3}\right\}\right)=$ $\frac{9}{4}$. If $G\left[e_{1}, e_{2}, e_{3}\right]$ is $S_{4}$, then $H\left(K_{5}-\left\{e_{1}, e_{2}, e_{3}\right\}\right)=\frac{79}{35}$. If $G\left[e_{1}, e_{2}, e_{3}\right]$ is a path, then $H\left(K_{5}-\left\{e_{1}, e_{2}, e_{3}\right\}\right)=\frac{83}{35}$. If $G\left[e_{1}, e_{2}, e_{3}\right]$ is not connected $\left(G\left[e_{1}, e_{2}, e_{3}\right]\right.$ is $\left.K_{2} \cup P_{3}\right)$, then $H\left(K_{5}-\left\{e_{1}, e_{2}, e_{3}\right\}\right)=\frac{37}{15}$. Thus $H\left(K_{5}-\left\{e_{1}, e_{2}, e_{3}\right\}\right) \geq \frac{9}{4}$ and from Lemma 2.1 we obtain $q\left(K_{5}-\left\{e_{1}, e_{2}, e_{3}\right\}\right) \leq \frac{13}{2}$, which gives

$$
\frac{q\left(K_{5}-\left\{e_{1}, e_{2}, e_{3}\right\}\right)}{H\left(K_{5}-\left\{e_{1}, e_{2}, e_{3}\right\}\right)} \leq \frac{26}{9}<\frac{16}{5} .
$$

If $m=4,5$ or 6 , it can be proved similarly that $\frac{q(G)}{H(G)}<\frac{16}{5}$.

From Theorems 3.6 and 3.7, and Corollaries 3.1 and 3.2 we obtain our main result (Theorem 1.1).

Acknowledgements. The work of T. Vetrík was supported by the National Research Foundation of South Africa; grant numbers: 112122, 90793.

\section{REFERENCES}

[1] M. Aouchiche, P. Hansen and M. Zheng, Variable neighborhood search for extremal graphs. 18. Conjectures and results about Randić index, MATCH Commun. Math. Comput. Chem. 56 (2006), 541-550.

[2] M. Aouchiche, P. Hansen and M. Zheng, Variable neighborhood search for extremal graphs. 19. Further conjectures and results about the Randić index, MATCH Commun. Math. Comput. Chem. 58 (2007), 83-102.

[3] B. Bollobás and P. Erdős, Graphs of extremal weights, Ars Comb. 50 (1998), 225-233.

[4] R. A. Brualdi and E. S. Solheid, On the spectral radius of connected graphs, Publ. Inst. Math. (Beograd) 39 (1984), 45-54.

[5] H. Deng, S. Balachandran and S. K. Ayyaswamy, On two conjectures of Randić index and the largest signless Laplacian eigenvalue of graphs, J. Math. Anal. Appl. 411 (2014), 196-200.

[6] H. Deng, S. Balachandran, S. K. Ayyaswamy and Y. B. Venkatakrishnan, On the harmonic index and the chromatic number of a graph, Discrete Appl. Math. 161 (2013), 2740-2744.

[7] H. Deng, S. Balachandran, S. K. Ayyaswamy and Y. B. Venkatakrishnan, On harmonic indices of trees, unicyclic graphs and bicyclic graphs, Ars Comb. 130 (2017), 239-248.

[8] S. Fajtlowicz, On conjectures of Graffiti II, in: Combinatorics, graph theory, and computing, Proceedings of 18th Southeast Conference in Boca Raton, Florida, Congr. Numerantium 60, 1987, 189-197.

[9] O. Favaron, M. Mahéo and J.-F. Saclé, Some eigenvalue properties in graphs (conjectures of Graffiti - II), Discrete Math. 111 (1993), 197-220.

[10] L. Feng and G. Yu, On three conjectures involving the signless Laplacian spectral radius of graphs, Publ. Inst. Math. (N.S.) 85 (2009), 35-38.

[11] P. Hansen and C. Lucas, Bounds and conjectures for the signless Laplacian index of graphs, Linear Algebra Appl. 432 (2010), 3319-3336. 
[12] P. Hansen and D. Vukicević, Variable neighborhood search for extremal graphs. 23. On the Randić index and the chromatic number, Discrete Math. 309 (2009), 4228-4234.

[13] Y. Hong, A bound on the spectral radius of graphs, Linear Algebra Appl. 108 (1988), 135-139.

[14] $\mathrm{Y} . \mathrm{Hu}$ and $\mathrm{X}$. Zhou, On the harmonic index of the unicyclic and bicyclic graphs, WSEAS Transactions on Mathematics 12 (2013), 716-726.

[15] B. Ning and X. Peng, The Randić index and signless Laplacian spectral radius of graphs, Discrete Math. 342(3) (2019), 643-653.

[16] M. Randić, On characterization of molecular branching, J. Amer. Chem. Soc. 97 (1975), 66096615.

[17] L. Zhong, The harmonic index on unicyclic graphs, Ars Combin. 104 (2012), 261-269.

${ }^{1}$ College of Mathematics and Statistics,

Hunan Normal University,

Changsha, China

Email address: hydeng@hunnu.edu.cn

${ }^{2}$ Department of Mathematics and Applied Mathematics, University of the Free State,

Bloemfontein, South AfricA

Email address: vetrikt@ufs.ac.za

${ }^{3}$ Department of Mathematics, School of Arts, Sciences and Humanities, SASTRA DEEMED UNIVERSITY,

ThanjaVur, IndiA

Email address: bala_maths@rediffmail.com 